\title{
Risk Predictive Factors to Convert Laparoscopic Cholecystectomy into Other Procedures
}

\author{
Eldo E Frezza* \\ Eastern New Mexico University; Roswell NM, Mexico
}

*Corresponding author: Eldo E Frezza, Eastern New Mexico University; Roswell NM, Mexico

\begin{abstract}
Laparoscopic cholecystectomy (LC) is nowadays the procedure of choice for cholecystitis. The intraoperative finding can make this procedure quite tricky such as dense adhesions at calot's triangle, fibrotic and contracted gallbladder, acutely inflamed or gangrenous gallbladder, cholcystoenteric fistula, etc. There are also risk factors which make laparoscopic surgery difficult like old age, male sex, obesity, previous abdominal surgery, thickened gallbladder wall, distended gallbladder, pericholecystic fluid collection, impacted stone, etc.

Methods: This is a one cohort retrospective review of patients admitted to the hospital with acute cholecystitis who during LC were converted to intraoperative cholecystostomy tube placement (CCT) or to open cholecystectomy (OC). Preoperative risk factors to predict difficult cholecystectomy were evaluated.

Results: Medical records were reviewed retrospectively from January 2010 through December 2016. IRB approval was obtained. LC was performed in 556 cases between 2010-2016, with 56 (10\%) conversion: 39 CCT and 17 OC. The highest reason for conversion are Perioperative fluid around the gallbladder before surgery on the ultrasound (10\%), preoperative thickness of the gallbladder (9\%), Impacted stones (7\%) are the predicting factor that have more changes to turn the LC into a different surgical approach. These three parameters are followed by Prior Hospitalization (3\%) and presence of abdominal scar (5\%). Essential factors to make a problematic surgery were postoperative perivasculitis (2\%), obesity (8\%), difficult liver mobilization (1\%), acute and scleroatrophic cholecystitis (1\%), "porcelain gallbladder" (2\%). Causes of bleeding during our operation were: cirrhosis $(2 \%)$, accidental adhesion tearing $(0.5 \%)$.
\end{abstract}

Conclusion: Problematic LC can be diagnoses before the surgery and make the OR team ready for different surgical approach.

Keywords: Laparoscopic Cholecystectomy; Open Cholecystectomy; Cholecystostomy Tube; Difficult Cholecystectomy; Predictive Factors for Difficult Surgery.

\section{Introduction}

Laparoscopic cholecystectomy (LC) is nowadays the procedure of choice for cholecystitis [1]. The intraoperative finding can make this procedure quite trickysuch as denseadhesions atcalot's triangle, fibrotic and contracted gallbladder, acutely inflamed or gangrenous gallbladder, cholcystoenteric fistula, etc. [2]. There are also risk factors which make laparoscopic surgery difficult like old age, male sex, obesity, previous abdominal surgery, thickened gallbladder wall, distended gallbladder, pericholecystic fluid collection, impacted stone, etc. [3]. A cholecystostomy is an opening made in the gallbladder, to place a tube for drainage. John Stough Bobbs, in 1867, was the first to described it $[4,5]$. It has been used in 1) person is ill, and 2) to defer cholecystectomy [6]. Todd Baron and Mark Topazian in 2007 place the first percutaneous Cholecustostomy Tube using ultrasound guidance [7]. The role of Cholecystostomy tube (CCT) is controversial in current surgical practice [8]. In critically ill patients, cholecystostomy tubes should remain in place until the patient is deemed medically suitable to undergo cholecystectomy. Removal of the cholecystostomy tube without subsequent cholecystectomy was reported associated with a high incidence of recurrences. [9] Attempts to predict intraoperative difficulties was described and included, palpable gallbladder, pericholecystic fluid, male more than female incidence, etc. [11], but still is not an $100 \%$ given all patients are different. Technical and tactical solutions to deal with complicated cholecystitis surgery were reported [10] but cannot 
be always applicable. Our study is based on the assumption that difficulty cholecystectomy can be defined before the surgery and give the opportunity to the surgical team to prepare for alternate surgeries option like Open Cholecystectomy (OC) or intraoperative Cholecystostomy Tube placement (CTT).

\section{Methods}

This is a retrospective review of patients admitted to the hospital who were diagnosed with acute cholecystitis who underwent an initial laparoscopic Cholecystectomy. The study was designed to find those patients who were converted in other surgery than LLC and check if the preoperative work out was predictive of failure of LLC. The Cohort taken in consideration were those who converted into Cholecystostomy Tube Placement (CCT) or to open cholecystectomy (OC). Medical records were reviewed for demographic data, diagnoses, imaging, complications, and outcomes: from January 2010 through December 2016 from the same surgeon. IRB approval was obtained.

a) Inclusion Criteria: All patients who underwent LC from January 2010 to December 2016 were included in the study.

b) Exclusion Criteria:

i. Laparoscopic cholecystectomy performed with other laparoscopic intervention in the same setting.

ii. Laparoscopic cholecystectomy with Common Bile Duct (CBD) exploration.

iii. Absolute contraindications to LC like cardiovascular, pulmonary disease, coagulopathies, and end-stage liver disease. Demographic symptoms sings of presentations were evaluated to find if those were impacting on our surgeries and addresses the activities of the cholecystectomy. The evaluated risk were the following: history os hospitalization, palpable gallbladder, thicken gallbladder, peri-cholecystitis fluid, impacted stones at the neck, abdominal scar. The characteristic of the patients was reported in Table 1.

\section{Pre And Intraoperative}

A detailed proforma was in place before the surgery to record information regarding patient history, physical examination, laboratory parameters, ultrasonography (USG) findings and intraoperative details.

\section{Operative Technique}

After obtaining an informed consent including an option for CCT and $\mathrm{OC}$ the patient was taken to the operating room placed under general anesthesia and prep in the usual fashion. The first incision was done in the left upper quadrant with a knife and a trocar, and a camera was advanced through the tissue under direct vision. Once in the abdomen, we obtained a pneumoperitoneum of $15 \mathrm{mmHg}$. We place 2 five $\mathrm{mm}$ trocars in the right upper quadrant, one at the level of the belly button of $5 \mathrm{~mm}$. The initial trocar was switched to a $12 \mathrm{~mm}$ trocars. Evaluation of the Right upper quadrant and the gallbladder was made.

\section{Critical Factors}

The crititical factor evaluated to continue the LC or turned into CCT or OC: 1) a change of the color of the gallbladder (green etc), 2) multiple adhesion which could not be taken out, 3) inability to grab the gallbladder after aspirating with the needle, 4) failure to see after the body of the gallbladder and define the neck of the gallbladder without good vision of the area of the common bile duct.

\section{CCT}

The fundus of the gallbladder was open with the Bovie. The fluid was aspirated, and the stones inside in the gallbladder were taken out by grasping with a laparoscopic Babcock after all the stones were cleaned and placed one by one in a separate bag inserted in the abdomen. The bag was closed. We then whased the gallbladder with saline, which also helps to mobilize hidden stones. The camera was then advance inside the gallbladder and evaluated from inside visualize the cystic duct. Once we know they there no other stones obstructing, a 2/0 silk purse string was placed at the fundus opening. With a separate incision, a Foley 18 French was inserted in the abdomen and the tip placed inside the gallbladder. The purse string was tied, and the balloon of the Foley was filled with seven ml. of saline. A Jackson Prat was placed at the liver fossa and secure with a 2/0 nylon to the skin. Same suture was used to secure the CCT to the skin. as we did to achieve the Foley now new cholecystostomy tube. The CCT was connected to a Foley bag and left on gravity. Given the difficult to have a real CCT, we usually use a Foley 18 French as CCT tube. We wash the abdomen and close the trocars with 4/0 monocryl and dermabond. After surgery, the patient was allowed to advance the diet and walk. Most of the patients were discharged within 36 hours with home health.

\section{OC}

If the CCT was not possible with a knife, we made a subcostal incision. The incision was then taken down with the bouvie while separating the muscle. Once in the abdomen, we close the gas insufflation. Few laps were placed on the stomach, duodenum and colon side. The gallbladder was grasped with a kelly clamp and dissected with bouvie from the liver. Once at the neck of the gallbladder was visualized the artery and the cystic duct, were dissected either between clips or with vascular staplers. Jackson Prat ten French drainage was placed in the liver fossa and secure to the skin. The wound was closed in layers with one vycril and stapler for the skin. The patient was allowed fluid, they were placed on PCA pump and discharge home with home health care within 4 days.

\section{Post OP Treatment of the CCT}

The tube was left on biliary bag drainage, Cholangiogram is ordered between week 4 and 6 . If no stones were found from the 
cholangiogram the tube was pulled out in the office otherwise redo surgery was scheduled.

\section{Results}

Table 1: Patient Characteristics.

\begin{tabular}{|c|c|}
\hline Category & Conversion \\
\hline Total patients & $56 / 556$ \\
\hline Age & $65+10$ \\
\hline Gender (M/F) & $34 / 22$ \\
\hline BMI & $49+10$ \\
\hline Abdominal Scar preop & $30 / 556(5 \%)$ \\
\hline Prior Hospitalization & $18(3 \%)$ \\
\hline Preop Palpable GB & $7(1 \%)$ \\
\hline Preop Thick GB wall & $50(9 \%)$ \\
\hline Preop Peri GB fluid & $56(10 \%)$ \\
\hline Impacted stones & $37(7 \%)$ \\
\hline postoperative perivasculitis & $10(2 \%)$ \\
\hline obesity & $47(8 \%)$ \\
\hline difficult liver mobilization & $6(1 \%)$ \\
\hline sclero-atrophic cholecystitis & $8(1 \%)$ \\
\hline "porcelain gallbladder" & $11(2 \%)$ \\
\hline cirrhosis & $10(2 \%)$ \\
\hline adhesion tearing & $3(0.5 \%)$ \\
\hline Post op. Hospital stay & $3+1$ \\
\hline Post op pain severe $(10 / 10)$ & $12 / 56(17 \%)$ \\
\hline Post op Seroma & $10 / 56(16 \%)$ \\
\hline Intra-Op. Blood Loss ml. & $170+40$ \\
\hline Require more surgery & $1 / 56(1 \%)$ \\
\hline
\end{tabular}

556 cases were performed between 2010-2016 by the same surgeon, Total 56 patients (10\%) who match our criteria were converted: 39 CCT and 17 OC. The surgery was performed by the same surgeon in different hospitals. Mean intraoperative time was $51 \pm 26 \mathrm{~min}$ (range $27-77 \mathrm{~min}$ ) in CCT and $53 \pm 28 \mathrm{~min}$ (range 25-81 min) in OC. Postoperative hospital stay was $1.4 \pm 0.4$ days in CCT and $4 \pm 1$ in OC $(\mathrm{p}<0.05)$. The operative data, time bleeding and postoperative hospital stay, seromas incidence were collected and reported in Table 1. The following comorbidities were founded: cardiovascular disease (20 patients), respiratory failure (10 patients). At 30 days, the morbidity associated with the CCT itself was $4 \%$ while OC was $70 \%$. Of the patients who underwent CCT only one $(10 \%)$ underwent LC after 30 days. Perioperative fluid around the gallbladder before surgery on the ultrasound $(10 \%)$, preoperative thickness of the gallbladder (9\%), Impacted stones (7\%) are the predicting factor that have more changes to turn the LC into a different surgical approach. These three parameters are followed by Prior Hospitalization (3\%) and presence of abdominal scar (5\%) (Table 1). Essential factors to make a problematic surgery were: postoperative perivasculitis $(2 \%)$, obesity $(8 \%)$, difficult liver mobilization (1\%), acute and scleroatrophic cholecystitis (1\%), "porcelain gallbladder" (2\%). Causes of bleeding during our operation were: cirrhosis (2\%), accidental adhesion tearing $(0.5 \%)$ (Table 1).

\section{Discussion}

With the help of accurate prediction, the high-risk patient may be informed beforehand regarding probability of conversion to OC or CCT. This discuss will also help the surgeon and the OR team to prepare the alternative surgeries. Surgeons should be aware of the possible complications that may arise in high-risk patients.

\section{Risk Predictors Factors}

Male sex makes surgery difficult as being reported in studies [10-12]. Conversion rate and significantly higher mortality $[13,8]$ and found to be a significant factor. Subtotal cholecystectomy, antegrade and fundus first techniques which is now being more commonly done during LC were associated with lower complications and conversion rate. Other risk factors for difficulty surgery are reported as increased age, acute and thick wall chronic cholecystitis, wide and short cystic duct, cholecyst digestive fistula, previous upper abdominal surgery, obesity, liver cirrhosis, anatomic variation, cholangiocarcinoma, and low surgeon's caseload [14]. Although decompression and drainage of the gallbladder through a radiological placed cholecystostomy tube may be used as a temporary treatment of acute cholecystitis in ill population, there is still some debate about the management of the tube and the subsequent need for a cholecystectomy. Other authors reported 105 patients, 12 (11.4\%) required conversion to open cholecystectomy. They pointed out that their significant predictors of conversion were body mass index $>30 \mathrm{Kg} / \mathrm{m}^{2}$, male gender, history of acute cholecystitis or acute pancreatitis, the recent history of upper abdominal surgery, and gallbladder wall thickness exceeding $3 \mathrm{~mm}$ [15]. Thickened gallbladder wall is an ultrasonographic finding of acute cholecystitis, and it was a significant factor in previous studies [16-18]. James Majeski [16], showed that a preoperative gallbladder ultrasound evaluation with a thick gallbladder wall ( $>3 \mathrm{~mm}$ ) and calculi, is a clinical warning for a problematic laparoscopic cholecystectomy procedure which may require conversion to an open cholecystectomy procedure [19].

But Carmody concluded that detailed preoperative ultrasound evaluation of the gallbladder in patients destined for laparoscopic cholecystectomy is of little value in screening for difficult or unsuitable cases. They found that there were no ultrasound features that can differentiate between the unsuccessful, confusing, or uneventful laparoscopic cholecystectomy [20]. In our study thickened gallbladder wall was present in all patients and outcome was found to be dependent on this variable by chi-square test ( $p$ $=0.001$ ), and logistic regression analysis also ascertained the significance of this factor for prediction $(p=0.005)$. Pericholecystic fluid is an ultrasonographic finding of acute cholecystitis. This was found to be a significant factor in our study ( $p=0.939$ ), as well as palpable gallbladder $(\mathrm{p}=0.05)$. Therefore, we agree with 
Randhawa [21] who also reported that presence of palpable gallbladder has a significant bearing on define difficult surgery. Difficulty in gallbladder grasping was associated significantly with the conversion. A distended gallbladder or a gallbladder filled with stones is not easily grasped because it tends to slip away. Presence of inflammation around the gallbladder makes the wall friable and edematous, thus posing problems. These data were reported by Singh [22] who also found a significant association between difficulties in grasping a distended gallbladder and pericholecystic inflammation. Lal [23] have identified that presence of large stones in the gallbladder neck leads to distention and difficulty in grasping.

\section{Cholecystostomy}

Percutaneous Cholecystectomy (PCCT) is primarily indicated for accessing the gallbladder to manage cholecystitis or to serve as a portal to remove or dissolve gallstones [24,25]. In the current literature and clinical practice, surgeon and internal medicine physician continue to recommend PCCT as an alternative to surgical cholecystectomy in patients with acute cholecystitis deemed poor surgical candidates. This trend is mainly based on retrospective studies $[24,25,9]$ and anecdotal clinical experience, which result in an inconsistent and unsupported utilization of PCCT. The recommendation of PCCT over surgical alternatives will continue to be based mostly on clinical intuition until randomized, controlled trials answer a series of questions regarding the treatment of acute cholecystitis [8]. If surgical options under general anesthesia can be avoided by a fast, simple, low-risk procedure under conscious sedation in any patient, it stands to reason that that procedure should become the new primary treatment option. Controversy and confusion over the application of PCCT raise a critical question: Does the existing, albeit insufficient, literature support the potential use of PCCT as a first-line and potentially definitive therapy for any cases of acute cholecystitis? PCCT should be still considered in a critical ill patient who cannot stand general anesthesia. Some other authors were close to our concept and tried to dissolve the stones to avoid another surgery by placing CCT laparoscopically.

Authors have employed the cholecystostomy tract to facilitate removal of gallstones by basket extraction [26] dissolution with bile acids, and destruction and retrieval with shock-wave lithotripsy [27,28,29]. Retrospective studies have demonstrated a gallstone recurrence rate of $\sim 10$ to $30 \%$ per year and a symptomatic recurrence rate requiring repeat treatment of $\sim 6$ to $18 \%$ per year. Stone removal can be repeated as needed, but the high rate of symptomatic recurrence and the risks and consequences of recurrent acute cholecystitis may limit the future of this option as a definitive treatment. With nowadays improved laparoscopic technique the conversion rate should be minimal in our experience is only $10 \%$. The highest reason for conversion are Perioperative fluid around the gallbladder before surgery on the ultrasound (10\%), preoperative thickness of the gallbladder (9\%), Impacted stones $(7 \%)$ are the predicting factor that have more changes to turn the LC into a different surgical approach. These three parameters are followed by Prior Hospitalization (3\%) and presence of abdominal scar (5\%) (Table 1) and made high risk for performing another surgery but LLC. Other factor whoch can predict problematic surgery were: postoperative perivasculitis (2\%), obesity (8\%), difficult liver mobilization (1\%), acute and scleroatrophic cholecystitis (1\%), "porcelain gallbladder" (2\%). Causes of bleeding during our operation were: cirrhosis (2\%), accidental adhesion tearing $(0.5 \%)$ (Table 1$)$.

\section{Conclusion}

Problematic LC can be diagnoses before the surgery and make the OR team ready for different surgical approach. Conversion should be kept less than $20 \%$ of the cases in out experience was $10 \%$. PCCT should be still considered in a critically ill patient who cannot stand general anesthesia.

\section{References}

1. VH Le, DE Smith, BL Johnson (2012) Conversion of laparoscopic to open cholecystectomy in the current era of laparoscopic surgery. Am Surg 78 (12): 1392-1395

2. K Singh, A Ohri (2006) Difficult laparoscopic cholecystectomy: A large series from north India. Ind J Surg 68(4): 205-208.

3. NA Abdel Baki, MA Motawei, KE Soliman, AM Farouk (2006) Preoperative prediction of difficult laparoscopic cholecystectomy using clinical and ultrasonographic parameters. JMRI 27(3): 102-107.

4. Kelly, Howard A Burrage, Walter L (1920) Bobbs, John Stough. American Medical Biographies. Baltimore: The Norman, Remington Company.

5. (1901) Bulletin of the Johns Hopkins Hospital, Baltimore: The Johns Hopkins Press 12(249).

6. Bakkaloglu H, Yanar H, Guloglu R (2006) Ultrasound-guided percutaneous cholecystostomy in high-risk patients for surgical intervention. World J Gastroenterol 12(44): 7179-7182.

7. Baron TH Topazian (2007) Endoscopic transduodenal drainage of the gallbladder: implications for endoluminal treatment of gallbladder disease. Gastrointest Endosc 65(4): 735-737.

8. Jonathan M Lorenz (2011) Evaluating the Controversial Role of Cholecystostomy in Current Clinical Practice. Semin Intervent Radiol 28(4): 444-449.

9. Morse BC, Smith JB, Lawdahl RB, Roettger RH (2010) Management of acute cholecystitis in critically ill patients: a contemporary role for cholecystostomy and subsequent cholecystectomy. Am Surg 76(7): 708712 .

10. Palade R, Vasile D, Grigoriu M, Roman H, Caplan I (1997) Difficult laparoscopic cholecystectomy Chirurgia 92(2): 87-92.

11. Mittalgodu Anantha Krishna Murthy Vivek, Alfred Joseph Augustine, and Ranjith Rao (2014) A comprehensive predictive scoring method for difficult laparoscopic cholecystectomy. J Minim Access Surg 10(2): 6267.

12. HH Lein, CS Huang (2002) Male gender: a risk factor for severe symptomatic cholelithiasis World J Surg 26: 598-601.

13. L Bouarfa, A Schneider, H Feussner, N Navab, HU Lemke, et al. (2011) Prediction of intraoperative complexity from preoperative patient data for laparoscopic cholecystectomy Artif Intell Med 52(3): 169-176.

14. Hussain A (2011) Difficult laparoscopic cholecystectomy: Current evidence and strategies of management. Surg Laparosc Endosc Percutan Tech 21(4): 211-217.

15. Nachnani J, (2005) Pre-operative prediction of difficult laparoscopic cholecystectomy using clinical and ultrasonographic parameters. Indian 
Journal of Gastroenterology: Official Journal of the Indian Society of Gastroenterology 24(1): 16-18.

16. J Majeski (2007) Significance of preoperative ultrasound measurement of gallbladder wall thickness Am Surg 73(9): 926-929.

17. M Rosen, F Brody, J Ponsky (2002) Predictive factors for conversion of laparoscopic cholecystectomy. Am J Surg 184(3): 254-258.

18. C Simopoulos, A Polychronidis, S Botaitis, S Perente, M Pitiakoudis (2005) Laparoscopic cholecystectomy in obese patients. Obes Surg 15(2): 243-246.

19. SS Daradkeh,ZSuwan, M Abu Khalaf(1998) Preoperative ultrasonography and prediction of difficulties in laparoscopic cholecystectomy. World Surg 22: 75-77.

20. E Carmody, AM Arenson, S Hanna (1994) Failed or difficult laparoscopic cholecystectomy: can preoperative ultrasonography identify potential problems. J Clin Ultrasound 22(6): 391-396.

21. Randhawa JS, Pujahari AK (2009) Preoperative prediction of difficult lap chole: a scoring method. Indian J Surg 71: 198-201.

22. Singh K, Ohri A (2006) Difficult laparoscopic cholecystectomy: A large series from north India. Indian J Surg 68: 205-208.

This work is licensed under Creative

Commons Attribution 4.0 License

To Submit Your Article Click Here:

Submit Article

DOI: $10.32474 /$ CTGH.2019.02.000128

23. Lal P, Agarwal PN, Malik VK, Chakravarti AL (2002) A difficultlaparoscopic cholecystectomy that requires conversion to open procedure can be predicted by preoperative ultrasonography. JSLS 6: 59-63.

24. Courtois C S, Picus DD, Hicks ME (1996) Percutaneous gallstone removal: long-term follow-up. J Vasc Interv Radiol 7(2): 229-234.

25. Petroni ML, Jazrawi R P, Pazzi P (2000) The British-Italian Gallstone Study Group Risk factors for the development of gallstone recurrence following medical dissolution. Eur J Gastroenterol Hepatol 12(6): 695700 .

26. Carrilho Ribeiro L, Pinto Correia A, Velosa J, de Moura MC (2000) Longterm gallbladder stone recurrence and risk factors after successful lithotripsy. Eur J Gastroenterol Hepatol 12(2): 209-215.

27. Lazăr F, Duţă C (2001) Difficult laparoscopic cholecystectomy. Chirurgia; 1990 96(3): 269-276.

28. NikhilGupta, GyanRanjanb, MPArorab, BinitaGoswamic, et al. (2013) Validation of a scoring system to predict difficult laparoscopic cholecystectomy. International Journal of Surgery 11(9): 1002-1006.

29. Nicole Cherng BS, Elan T Witkowski, Erica B Sneider, Jason T Wiseman, Joanne Lewis, et al. (2012) Use of Cholecystostomy Tubes in the Management of Patients with Primary Diagnosis of Acute Cholecystitis. JACS 214(2): 196-201.

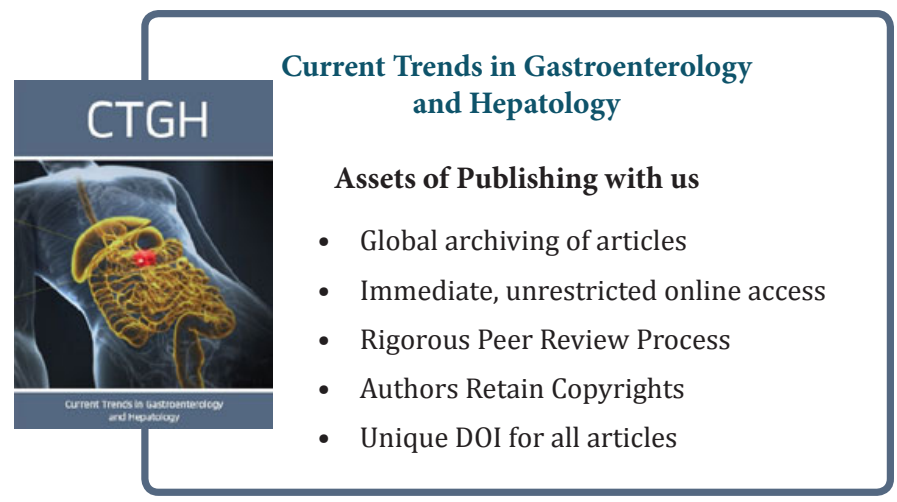

